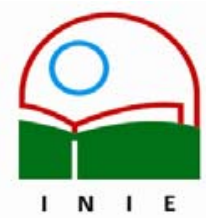

Actualidades Investigativas en Educación

Revista Electrónica publicada por el

Instituto de Investigación en Educación

Universidad de Costa Rica

ISSN 1409-4703

http://revista.inie.ucr.ac.cr

COSTA RICA

\title{
MARCO REFERENCIAL PARA EL ANÁLISIS DE LOS PROGRAMAS DE ENSEÑANZA MEDIA EN EL ÁREA DE FÍSICA Y DE QUÍMICA PROPUESTOS POR EL MINISTERIO DE EDUCACIÓN PÚBLICA COSTARRICENSE
}

\author{
REFERENCIAL FRAME WORK FOR THE ANALYSIS OF THE THIRD AND FOURTH \\ CYCLES STUDY PROGRAMS IN THE PHYSICS AND CHEMISTRY FIELDS PROPOSED \\ BY THE COSTA RICAN PUBLIC EDUCATION MINISTRY
}

\section{Volumen 8, Número 3}

pp. $1-19$

Este número se publicó el 15 de diciembre 2008

Leonardo Herrera Vargas

Yeudin Artavia Herrera

La revista está indexada en los directorios:

LATINDEX, REDALYC, IRESIE, CLASE, DIALNET, DOAJ, E-REVIST@S,

La revista está incluida en los sitios:

REDIE, RINACE, OEI, MAESTROTECA, HUASCARAN

Los contenidos de este artículo están bajo una licencia Creative Commons 


\title{
MARCO REFERENCIAL PARA EL ANÁLISIS DE LOS PROGRAMAS DE ENSEÑANZA MEDIA EN EL ÁREA DE FÍSICA Y DE QUÍMICA PROPUESTOS POR EL MINISTERIO DE EDUCACIÓN PÚBLICA COSTARRICENSE

\author{
REFERENCIAL FRAME WORK FOR THE ANALYSIS OF THE THIRD AND FOURTH \\ BY THE COSTA RICAN PUBLIC EDUCATION MINISTRY
} \\ CYCLES STUDY PROGRAMS IN THE PHYSICS AND CHEMISTRY FIELDS PROPOSED
}

\section{Leonardo Herrera Vargas ${ }^{1}$ \\ Yeudin Artavia Herrera ${ }^{2}$}

Resumen: Este artículo presenta el marco referencial para el análisis de los programas de estudio propuestos por el Ministerio de Educación Pública (MEP) en la enseñanza de la Física y la Química para la educación diversificada. Se considera el aporte que el MEP ofrece a los docentes de Física y Química, desde su posición de ente controlador de la educación costarricense. Así como el aporte de diversos autores que ofrecen variadas concepciones sobre la temática de enseñanza de la ciencia en general y de la Física y la Química en particular.

Palabras claves: ENSEÑANZA DE LA FÍSICA, ENSEÑANZA DE LA QUÍMICA, MODIFICACIONES CURRICULARES, PROGRAMAS DE ESTUDIO, COSTA RICA

\begin{abstract}
This article presents the referencial frame work for the analysis of the study programs proposed by the Public Education Ministry (MEP) for the teaching of Physics and Chemistry in the third and fourth cicles. The contribution given to the Physics and Chemistry teachers by the Ministry of education has been taken into consideration since it's the controlling entity in Costa Rican education. Besides, relevant ideas from several authors who have a variety of concepts about science teaching and Physics and Chemistry specifically were used to support the research.
\end{abstract}

Key words: PHYSICS TEACHING. CHEMISTRY TEACHING, CURRICULAR MODIFICATIONS, STUDY PROGRAMS, COSTA RICA

\footnotetext{
${ }^{1}$ Licenciado en la Enseñanza de la Física, Universidad de Costa Rica. Actualmente labora para la Escuela de Física de la UCR y Planetario de la Ciudad de San José de la UCR.
}

Dirección electrónica: LEONARDO.HERRERA@ucr.ac.cr

${ }^{2}$ Licenciado en la Enseñanza de la Química, Universidad de Costa Rica. Actualmente trabaja para el Ministerio de Educación Pública, CINDEA Alberto Manuel Brenes.

Dirección electrónica: yartavia82@gmail.com

Artículo recibido: 3 de agosto, 2008

Aprobado: 11 de noviembre, 2008 


\section{Introducción}

La sociedad actual ha logrado un nivel de vida relativamente elevado y desea mantenerlo y mejorarlo. La seguridad nacional de cada país, la calidad de vida, las condiciones sanitarias y algunas otras áreas que afectan la sociedad se han obtenido mediante el desarrollo de la ciencia. Esta se ha utilizado para crear terribles armas destructivas, para construir máquinas y desarrollar procesos que perfeccionan los bienes y servicios, y para comprender la índole de las enfermedades y el modo de controlarlas. En otras palabras, el desarrollo de la ciencia y la tecnología ha apoyado la calidad de vida pero también la ha destruido. La ciencia desempeña un papel dinámico en el progreso que el ser humano ha logrado. Sin embargo, es indispensable que la conquista científica de lo desconocido y la aplicación técnica de lo aprendido sea continua, para conservar lo que se ha logrado (Darrell, 1970).

La Física y en Química juegan un papel especial en el proceso de la enseñanza científica, pues muchos de los conceptos que son básicos para las otras disciplinas provienen de ellas. En consecuencia, ciertos conocimientos de estas ciencias deben ser introducidos en el proceso de enseñanza y aprendizaje tan tempranamente como sea posible, de manera que pueda disponerse de los elementos que son fundamentales para la construcción de nuevos conceptos en las otras disciplinas del plan de estudio (UNICEF, 1970, p.16).

La Física y la Química se consideran ciencias prácticas, donde el educando adquiere el conocimiento en la medida que experimenta, ya sea en un laboratorio o en situaciones de la vida cotidiana, de manera que se logre un aprendizaje significativo e integral, afín con otras áreas del conocimiento. La enseñanza de estas ciencias, y la educación en general, entendida como la actividad mediante la cual se produce y distribuye el conocimiento, asume en la actualidad coyuntural, una importancia inédita no sólo desde el punto de vista políticosocial, sino también de los contenidos de la socialización y su impacto en la inclusión o exclusión de amplios sectores de la población (Gurdián, 1999).

La educación adquiere importancia en el momento que es vista como un proceso continuo que ofrece al individuo conocimientos y la oportunidad de desarrollar habilidades y destrezas que le permiten tomar conciencia de sí mismo y de su entorno y de esta forma cumplir con su rol dentro de la sociedad. Además, la educación es vista como una herramienta de desarrollo 
individual y de grupo, un movilizador social capaz de cerrar las brechas entre las diferentes clases sociales.

La manera de poder plasmar los ideales y fines de la educación es mediante un diseño curricular que contemple los elementos didácticos y pedagógicos necesarios y adecuados para la población estudiantil. Tal es el caso de los programas de estudio, los cuales ofrecen la posibilidad de organizar, ejecutar y evaluar la información y el conocimiento de forma integral.

Este marco referencial nació como apoyo para conocer las modificaciones que han sufrido los programas de estudio de secundaria, en el área de la Física y de la Química, y reconociendo la influencia de la educación como un movilizador social capaz de cerrar las diferentes brechas de las clases sociales. Para ese estudio se escogió el período de 1980 a 1994 y la actual política educativa hacia el siglo XXI de 1995 al 2005, tomando en cuenta la vigencia de las dos políticas educativas mencionadas, se han seleccionado los programas de estudio del ciclo diversificado de Física y Química del periodo de 1980 al 2005. El Objetivo de la investigación que apoyó este marco referencial perseguía analizar la evolución que han sufrido los diferentes temas dentro de los programas de estudio, en el periodo mencionado.

\section{Ciencia y tecnología}

La ciencia, según Bunge (1984), debe concebirse como un sistema conceptual, compuesto de subsistemas, que son las ciencias especiales y las interdisciplinas tales como la biofísica y la psicobiología.

De acuerdo con Morales (2001), la tecnología toma el conocimiento y lo aplica, de forma tal que logra que hayan nuevos bienes y servicios. Aquí sería importante referirse, brevemente, al concepto de técnica, la cual se trató alguna vez en forma similar a la tecnología y está por lo tanto muy ligado a ella. Esta involucra, según el autor mencionado, actividades prácticas de prevención, operación o manejo y arreglo de los productos tecnológicos (bienes o servicios). 
La ciencia y la tecnología han sido siempre parte integral de la historia de la humanidad, contribuyendo con grandes conocimientos e inventos, los cuales han repercutido y formado el camino hacia nuevos estilos de vida de la sociedad.

\subsection{Los Orígenes y desarrollo de la ciencia: consideraciones históricas.}

Se suele considerar que el origen de la historia de la ciencia estuvo en el siglo XVIII, en el discurso elaborado por los científicos y filósofos sobre la revolución científica de los siglos $\mathrm{XVI}$ y XVII, aunque tuvo antecedentes previos.

Interesa para este estudio la evolución histórica de la Física y la Química

\subsubsection{La Física.}

Los relatos sobre la Física datan incluso desde el año 3000 a.C. con los antiguos babilonios y egipcios, los cuales estaban familiarizados con algunos de los principios fundamentales de la Física, relacionados con medidas de tierras y astronomía, además de los grandes indicios de la ingeniería, representados por las pirámides de Egipto. También los chinos estaban familiarizados con la brújula desde el año 1000 a.C.

Por otro lado, los griegos, en su mayoría filósofos, aportaron avances a la ciencia, como se observa en el periodo 700-150 a.C., caracterizado por el razonamiento deductivo más bien que inductivo, en su mayoría basado en observaciones más superficiales, en la curiosidad y en las especulaciones. Sin embargo, se ha dicho que fue Sócrates (469-399 a.C.) quién en la antigua Grecia sembró la semilla del método científico con sus razonamientos inductivos.

Aunque el desarrollo continuó hasta alrededor del año 1500 d.C., prácticamente ningún avance en la ciencia en general se hizo entre el año 50 a.C. y el 1550 d.C. Este período abarca el ascenso y la caída del Impero Romano, coincidiendo con la declinación de la cultura griega y con la invasión del antiguo mundo occidental por los bárbaros. Los romanos gradualmente adsorbieron la cultura griega, pero por el año 600 d.C. toda Europa había sido despojada de la oportunidad de valerse por sí misma, pues los romanos carecían de 
inclinación científica. Prácticamente todos los manuscritos griegos quedaron entre los árabes, quienes los preservaron para la posteridad.

Entre el año 700 y el 1100 de nuestra era, el restablecimiento de la instrucción y de la ciencia en particular, estuvo bajo la influencia y poderío de la Iglesia Católica. En este período los eruditos se adhirieron a los números griegos y romanos, dejando los números arábigos al comercio. Ésta fue otra razón que produjo un estancamiento de unos 1500 años, durante los cuales prácticamente no se hizo ningún avance en relación con la ciencia griega.

Alrededor del año 1500, la ciencia había justamente regresado al Estado. No obstante, la Iglesia había restablecido la ciencia en grandes universidades, que se encontraban bajo su control. En aquel tiempo no podía descubrirse la verdad sino que era dictada por la Iglesia y era un crimen de primer orden dudar de los puntos de vista de Aristóteles, estos no tomaban en cuenta que la experimentación es el mejor camino para establecer la verdad. Naturalmente, la ciencia degeneró bajo este sistema, puesto que las doctrinas eclesiásticas estaban tan entrelazadas con los hechos científicos, que estos no podían ser separados de los dictados religiosos.

Según los historiadores, a partir del siglo XVI tuvo lugar el período del nuevo amanecer, considerado como el nacimiento de la ciencia moderna. Esto fue consecuencia de una ruptura con la tradición y de una serie de realizaciones como la teoría heliocéntrica del Universo, las ideas fundamentales de la mecánica celeste moderna, la idea de la experimentación controlada, las tres leyes del movimiento y la ley de gravitación, entre otras, asociadas a los nombres de Copérnico, Galileo, Descartes, Tycho Brahe, Kepler, Gilbert, y Newton, entre otros. Ello habría dado lugar a un desarrollo continuo de los conocimientos, anclados en la razón y el método científico, bajo la forma de una acumulación o una evolución.

En los siglos XVIII y XIX la Física acumuló innumerables realizaciones. Se hicieron avances en los campos de la mecánica, el calor, la luz y la electricidad, en forma más o menos independiente, pero el trabajo de Newton proveyó el método de integrar todos estos conocimientos. Ciertamente el éxito de la Física newtoniana fue tan grande, que al final de 
este período parecía que todos los conocimientos físicos estaban a la vista. Casi todo parecía establecido, de aquí la designación de "período clásico".

La Física del siglo XIX se inició en el siglo XVII con la revolución de Descartes y Galileo contra el aristotelismo y se consolidó con Newton. Pero nuevas ideas, producto del avance en los conocimientos físicos, tomaron un puesto en el esquema cartesiano.

En el siglo XIX se siguió viendo el desarrollo de la ciencia moderna como la concreción de una nueva etapa de la historia de la humanidad, que dejando atrás la superstición y el mito mediante el seguro procedimiento de la observación, la experimentación y el establecimiento de leyes, había conducido a una Edad Científica.

Aunque las realizaciones del siglo XIX fueron destacadas, no debe creerse que se había llegado a la perfección, ya que existían algunas brechas y ciertas incoherencias que aún no estaban del todo claras.

En realidad, las dificultades se volvieron enormes cuando los físicos concentraron su atención en ellas, viéndose finalmente obligados a tomar puntos de vista completamente nuevos, como los utilizados en la teoría cuántica y en la relatividad. Esto no sucedió hasta que una serie de descubrimientos se realizaron: el aislamiento del electrón, el descubrimiento de la radiactividad y de los rayos $\mathrm{X}$, y la formulación del concepto de la estructura electrónica de la materia.

En el año de 1900 (siglo XX) se introduce la teoría cuántica propuesta por Planck, seguida por la teoría de la relatividad en 1905 propuesta por Einstein. Estas dos teorías fueron la base para muchos adelantos, porque dieron nuevos puntos de vista para explicar los fenómenos naturales.

En 1911 surge mucho interés por la Física atómica y durante la década de 1920 se hicieron grandes adelantos en la electrónica, en espectroscopía y en Física nuclear. Por otro lado en la década de 1930 hubo descubrimientos importantes como el del neutrón, la radiactividad artificial, la fisión del uranio, la cual fue el fundamento de la bomba atómica de 1945. 
Actualmente la Física progresa con tal rapidez que sólo podemos preguntarnos ¿Qué podrá descubrirse en el futuro y qué consecuencias tendrán esos descubrimientos en nuestra vida?

Sin embargo, hay que hacer también énfasis a una gran premisa respecto al desarrollo de la ciencia y la tecnología, la cual está dirigida al impacto social y sus secuelas que conlleva este progreso.

\subsubsection{La Química}

La Química como ciencia, se pretende decir que se inició mucho antes del siglo XVI, cuando los seres humanos empezaron a diferenciar las distintas sustancias y los cambios que experimentan bajo.

La Química se remonta a los tiempos prehistóricos, iniciando desde la antigüedad hasta el siglo VI d.C., donde el descubrimiento del fuego ofreció la primera oportunidad al hombre primitivo para efectuar reacciones u operaciones Químicas sin conciencia de la ciencia que aplicaba.

Sin embargo, es desde Mesopotamia y Egipto donde debemos buscar las más remotas huellas de la ciencia Química. La forma en que en estas culturas se asumió el pensamiento racional explica gran parte de lo que encontramos más adelante en la alquimia y en la Química.

Las civilizaciones de Egipto y Mesopotamia alcanzaron un alto grado de habilidad técnica en la metalurgia y en la fabricación del vidrio. En Egipto se han encontrado hornos para fundir hierro, que se remontan a una época de 3000 años a.C. y otros de bronce que corresponden a épocas del año 2500 a.C., mientras que en Mesopotamia se conocen bronces de épocas más antiguas, 3500 a 3000 años a.C. La aleación de cobre y estaño condujo en los países occidentales a la Edad del Bronce, que siguió a la Edad de la Piedra. En la antigüedad y en la Edad Media solo se conocía el azufre y el carbono, además de siete elementos que son: oro, plata, cobre, estaño, plomo, mercurio y hierro. 
También se han encontrado recetas o fórmulas para la fabricación de materiales vidriados que datan del siglo VII a.C. El ser humano común poseía también métodos de Química práctica más simples para preparar alimentos, ungüentos, aceites y licores, para teñir telas y otras actividades con que satisfacer sus necesidades cotidianas.

Las civilizaciones egipcia y mesopotámica desarrollaron una tecnología práctica, utilizando numerosos procesos y métodos químicos que pasaron luego a las generaciones posteriores.

Los pensadores griegos establecieron generalizaciones en las que yacen los principios de muchos conceptos modernos. Sus ideas constituyeron la base sobre la cual se edificaron las ciencias alejandrina, medieval y renacentista. Su explicación de la naturaleza afectó a todas las ramas de la alquimia y de la Química.

Los conocimientos y las destrezas en las aplicaciones técnicas que llevó consigo la vida industrial en la antigüedad no experimentaron ningún progreso rectilíneo después de la caída del mundo antiguo. La Grecia clásica había llegado paulatinamente por diversos contactos y mezclas con el Oriente, a un estado de cultura que se designa como helenismo y cuyo centro fue la ciudad de Alejandría, fundada en Egipto en el año 331 a. C. En Egipto hay que buscar el origen del rumbo de la investigación y de los esfuerzos peculiares cuyo conjunto se designa con el nombre de Alquimia, época que va desde el siglo IV hasta el siglo XVI. Esta es heredada a los árabes conquistadores a mediados del siglo VII y es llevada a España e Italia. Al comienzo del siglo XIII se extiende la alquimia en los países occidentales cristianos.

Entre los trabajos y objetivos de la alquimia se encontraban: transmutación de los metales (fabricación del oro) y búsqueda de la panacea, fermentos mágicos, el "gran elixir", el "magisterio", la "piedra de la sabiduría" ("piedra filosofal”). Estos escritos fueron envueltos en el mayor misterio y poco a poco dicha época se desliza paulatinamente entre mentiras y fraudes. Los últimos seguidores llegan hasta el siglo XIX.

En el periodo del siglo XV se sitúa el tránsito de la Edad Media a la Edad Moderna. Allí empieza también un nuevo capítulo en la historia del desenvolvimiento de las ciencias naturales, época conocida como la YatroQuímica, período que comprende desde comienzos del siglo XVI hasta mediados del siglo XVII. 
Esta época se caracterizó porque Paracelso puso la Química al servicio de la Medicina con el fin de explicar desde el punto de vista químico los procesos vitales. En vez de buscar, como los alquimistas, la panacea para curar todas las enfermedades, Paracelso quería hallar remedios curativos, combinaciones Químicas, especialmente sales metálicas. Entre los continuadores de Paracelso hubo charlatanes de mercado e investigadores serios. En el siglo XVI florece la técnica Química de Jorge Agrícola.

Todo lo que hasta aquí se había hecho en Química, se basó en supuestos y no se interrogaba a la naturaleza para establecer las propiedades de las sustancias.

La época de la Química, propiamente dicha, comienza desde mediados del siglo XVII, con el alemán Jungius y el inglés Boyle. Se reconoce como misión propia de la Química la investigación de las propiedades y de las transformaciones de las sustancias. La teoría del flogisto, fundada por Becher y Stahl, domina al poco tiempo toda la Química. Se aclara el concepto de elemento, pero son confusas las ideas de masa y del peso. Surge el período cualitativo de la Química.

Basándose en el descubrimiento del oxígeno por Scheele (1771) y Priestley (1974), Lavoiser desarrolla la nueva teoría de la oxidación, aclara los conceptos de masa y peso de las sustancias Químicas y funda como físico la época de la Química cuantitativa. Se da la fundación de la estequiometría por J. B. Richter (1792), de la teoría atómica moderna, por Dalton (1803), y se descubren varias leyes fundamentales. Aparece un nuevo desarrollo de la Química, especialmente bajo la dirección de Berzelieus. Con la obtención artificial de la urea por Wöhler (1828) da comienzo la Química orgánica sintética. Los trabajos de Liebieg para la investigación y la enseñanza, adquieren gran importancia. Existe una aclaración de las hipótesis y conceptos teóricos. Se desarrolla la Química Física, especialmente en Alemania y crecen con gran rapidez las distintas ramas de la investigación, con resultados en parte demoledores (la radiactividad entre otros).

\subsection{El impacto social de la ciencia}

El hablar de la ciencia y de su impacto social es un tema que no se excluye de la cultura, la cual según Taylor "es ese todo complejo que incluye conocimientos, creencias, arte, moral, Volumen 8, Número 3, Año 2008, ISSN 1409-4703 
leyes, costumbres y cualesquiera otras capacidades y hábitos adquiridos por el hombre como miembro de una sociedad" (Medina y otros, 2000), por lo tanto "la ciencia no puede dejar de ser cultura, ni de ser parte de la riqueza humanística del hombre". (CONICIT, 1978).

Las culturas evolucionan en la medida en que su desarrollo social, político, económico, educativo, científico y tecnológico experimenta transformaciones significativas, como respuesta a los cambios y variaciones que se dan en el ambiente, desde el punto de vista natural y social.

Esto se hacer notar en las innovaciones científicas y tecnológicas, tal como lo menciona Medina, al decir:

Mirando hacia atrás desde el umbral del siglo XXI, se hace evidente que las innovaciones tecnocientíficas han sido los factores fundamentales que han configurado las culturas propias. Han modelado decisivamente el conjunto de las formas de vida, los entornos tanto materiales como interpretativos y valorativos, las cosmovisiones, los modos de organización social, económica y política junto con el medio ambiente característicos de esa época. (Medina y otros, 2000)

A lo largo de la historia el ser humano ha ido creando e innovando instrumentos y aparatos, ya sea por necesidad o simplemente por entretenimiento, lo cierto es que la funcionalidad de cada uno de ellos le ha permitido a la humanidad crecer científica y tecnológicamente, de manera que le facilita al hombre su estilo de vida.

La ciencia y la tecnología han jugado un papel fundamental en la evolución social del ser humano y como tal en su formación intelectual y laboral. Es por esto que a la educación le compete contribuir con ello, facilitando una instrucción de carácter científico, orientada hacia una enseñanza de las ciencias de manera más integral y sana, para formar personas con mayor capacidad de criterio, análisis y comprensión, que sepan resolver los problemas de la sociedad y dar soluciones adecuadas según sus necesidades. 


\subsection{Importancia de la enseñanza de las ciencias}

Durante los últimos años, el progreso se presenta tan rápido que las técnicas nuevas, fueron consideradas descubrimientos revolucionarios hace poco tiempo. Estos descubrimientos, posiblemente tengan hoy un valor discutible y resulten mañana anticuados. Una nueva arma nos promete seguridad si está en nuestras manos; pero en las del enemigo nos pone frente a los complejos problemas de la defensa. La magnitud y variedad de máquinas que nos permiten utilizar los recursos energéticos a velocidades astronómicas también determinan que el descubrimiento de nuevos recursos energéticos sea un problema de primerísimo orden para que podamos sobrevivir. Las drogas maravillosas que nos protegen de un microbio mortal pueden dar lugar a otro mutante, del que ninguna droga maravillosa pueda ya protegernos.

Pero todos estos conocimientos científicos es necesario transmitirlos a las nuevas generaciones. De ahí la importancia de la enseñanza de las ciencias.

La adquisición del conocimiento científico, lejos de ser un producto espontáneo y natural de nuestra interacción con el mundo de los objetos, es una laboriosa construcción social, o mejor aún reconstrucción, que sólo podrá alcanzarse mediante una enseñanza eficaz que sepa afrontar las dificultades que ese aprendizaje plantea.

Para que el conocimiento sirva de base a la acción humana, la transformación del mundo, debe dar un paso difícil pero indispensable: ser comprensibles para la gente. Más aún, tiene que ser capaz de convertirse en algo más que conocimiento: debe ser creible volverse parte de nuestro sistema de creencias, y no ser simplemente sabidos. En otras palabras, tienen que pasar a ser parte de nuestra cultura. Por esas razones, la labor de la educación científica es lograr que los alumnos construyan en las aulas actitudes, procedimientos y conceptos que por sí mismos no lograrían elaborar en contextos cotidianos y que, siempre que estos conocimientos sean funcionales, los trasfieran a nuevos contextos y situaciones. De esta forma, el currículo de las ciencias, desarrollado mediante las actividades de aprendizaje y enseñanza, debe servir como una auténtica ayuda pedagógica, una vía para que el alumno acceda a formas de conocimiento que por sí mismas le serían ajenas o al menos muy distantes. 


\subsubsection{Las escuelas educan a los futuros ciudadanos de la sociedad tecnológica}

El desarrollo de la ciencia es obra de las personas. Los grandes descubrimientos científicos son fruto de los esfuerzos humanos para interpretar los hallazgos de sus investigaciones. Las aplicaciones técnicas de esos descubrimientos para ampliar el bienestar de la comunidad, también son obra del ser humano. Toda la ciencia es creación humana, y su futuro, en los dominios del progreso teórico y técnico, dependerá de la calidad y número de nuestros futuros científicos.

Nuestra necesidad de mano de obra con preparación científica es mayor que en cualquier otro período histórico. Pero, en proporción, son menos los estudiantes universitarios que hoy se preparan para carreras científicas. En los Estados Unidos muchos ciudadanos consideran esta situación como uno de los problemas más graves del país. Este hecho puede estar relacionado, con la situación que impera, por ejemplo, en el sistema educativo de España, ya que un estudio sobre la forma en que los profesores de ciencias de secundaria conciben la evaluación en ese país demostró que casi el 90\% de los profesores de Física y Química están convencidos de que en su materia una evaluación adecuada es aquella que "suspende" a la mitad de los alumnos. Si la mayoría aprueba tienden a creer que esa evaluación ha estado mal diseñada. Esto es muy crítico porque puede ser el motivo de que cada vez más estudiantes pierdan el interés o la motivación y desistan de optar por carreras científicas a nivel universitario (Darrel, 1970).

Esta misma situación puede ser también la realidad que se está viviendo en Costa Rica y quizás en el resto del mundo. Por eso, hay que buscar nuevas metas educativas dirigidas a desarrollar en los alumnos capacidades que les permiten afrontar los cambios culturales que se están produciendo no solo en la vida social, sino sobre todo en los perfiles profesionales y laborales y en la propia organización y distribución social del conocimiento.

En una democracia los ciudadanos deben tomar muchas decisiones. Entre ellas, las que conciernen al apoyo financiero de la investigación científica, ya sea por parte de corporaciones privadas o por organismos gubernamentales, que dependen en último término del público, que también decide como se deben manejar los recursos naturales. Los ciudadanos determinan las características de los programas sanitarios, nacionales y locales, al declarar en qué medida están dispuestos a contribuir y, por ende qué grado de eficacia y Volumen 8, Número 3, Año 2008, ISSN 1409-4703 
permanencia pueden alcanzar esos programas. Para que las decisiones que se tomen en este y otros asuntos sean inteligentes, es necesario contar con una ciudadanía provista de conciencia científica. Lograr ese nivel es responsabilidad de los centros educativos y, dentro de ellas, en especial, de la enseñanza científica. Como las instituciones educativas pertenecen al pueblo, esta es la autoridad final para decidir qué debe enseñarse y qué tipo de instalaciones deberán emplear para ello.

Sin embargo, la realidad no es así, se sabe que el estado es el que tiene control de todos los bienes y servicios públicos, y el deber de educar ciudadanos para que enfrenten una sociedad tecnológicamente activa. Para educarse se requiere de una gran inversión con que el pueblo no cuenta, ya que es necesario suministrar a las instituciones educativas equipos tecnológicos y aulas adecuadas para desarrollar el conocimiento, las habilidades y las destrezas tecnológicas en los estudiantes. Por otro lado, se debe realizar un currículo (para el cual no es consultado el pueblo) donde se considere cuáles son los objetivos, contenidos, procedimientos, evaluación, entre otros elementos, los cuales se espera que el alumno desarrolle, para que pueda tener las herramientas necesarias para enfrentarse ante una sociedad tecnológica.

\subsubsection{Las escuelas educan para facilitar la adaptación}

Desde que son concebidos, pasando por sus primeros meses de vida y luego por la infancia, la adolescencia, la edad adulta y la vejez, los seres humanos cambian siempre, en cuanto son organismos biológicos en funcionamiento. Cambian en su modo de sentir acerca de sí mismos y hacia los demás y el modo en que los trata la sociedad. En cada etapa de su desarrollo, el individuo debe satisfacer ciertas necesidades si aspira a lograr una relación favorable consigo mismo, con sus semejantes y con su medio. La identificación de esas necesidades y el examen del papel que debe desempeñar la educación para satisfacerlas, son factores que contribuyen a definir los objetivos, contenidos y métodos de todo programa educativo. En el caso de los adolescentes, una buena selección de contenidos y métodos científicos puede contribuir a satisfacer esas necesidades.

La gente joven posee variados intereses que es posible despertar y fomentar por medio de programas científicos debidamente planeados. Cuando entran a estudios de secundaria, muchos ya han revelado un talento especial que con la guía de un programa científico rico y 
estimulante los llevará luego a seguir carreras científicas. Todos los estudiantes de secundaria hallan, en los programas de ciencias, situaciones que se relacionan con sus problemas personales y sociales, una ayuda para formarse un concepto propio del papel decisivo que desempeña la ciencia en el moderno mundo tecnológico. Los jóvenes pueden tener experiencias científicas que les crean actitudes mentales y espíritu crítico, capaces de permitirles afrontar mejor los problemas y dar a estos las soluciones más adecuadas. Tales atributos son básicos para la solución inteligente de problemas personales y sociales y, por lo mismo, para una más eficiente educación cívica. Aunque la formación de esas actitudes y capacidades puede también integrarse a un programa de estudio sociológico, la ciencia puede aportar una contribución que es única.

En Costa Rica, el encargado de controlar los programas de cursos que enseñan esas ciencias es el Ministerio de Educación Publica, ente controlador del sistema educativo costarricense, cuya estructura administrativa presenta asesores por materia, lo que permite contar con un asesor nacional para cada ciencia. Esa estructura del MEP se ofrece en la siguiente temática.

\subsection{Programas de estudio.}

Los programas de estudio son de suma importancia para alcanzar los ideales educativos de una nación, que no solo corresponden a fines ideológicos o filosóficos, también representan un medio que aporta conocimientos y bases para el desarrollo de nuevas habilidades, destrezas y actitudes, en la formación de un individuo de provecho para el progreso del país. En Costa Rica, con los programas de estudio se pretende lograr en los educandos no solo un mayor nivel cognitivo, sino también una mejor formación en valores y actitudes, orientados bajo una política humanista, racionalista y constructivista, que conduzcan al individuo hacia su desarrollo integral.

En ente controlador utiliza su propio concepto de programa de estudio y sus componentes, y cuál es el enfoque u orientación que estos desempeñan en el área de la Física y de la Química. En el MEP se utiliza la siguiente definición:

Los PROGRAMAS DE ESTUDIO deben ser una guía relevante para el docente desde el punto de vista de la planificación de su trabajo de mediación en coherencia con los Volumen 8, Número 3, Año 2008, ISSN 1409-4703 
recursos didácticos, el proceso de evaluación y sus propias necesidades e intereses profesionales. Todo programa de estudio debe incluir objetivos, contenidos, experiencias y estrategias de mediación del aprendizaje y evaluación. (MEP, 1994)

Los componentes de estos programas se le ofrecen al docente con una estructura que es propia de cada materia y se presentan en columnas que ofrecen la siguiente información:

OBJETIVOS deben contemplar los contenidos, los procesos cognitivos y los valores que se persiguen realizar durante el ciclo lectivo en cuestión sobre la base del ciclo lectivo anterior, con una perspectiva de crecimiento y profundización hacia el ciclo lectivo venidero.

CONTENIDOS deben organizarse con base en conocimientos sólidos y rigurosos de cada disciplina; deben ser claros y autoexplicativos, consecuentes con la planificación de los objetivos.

EXPERIENCIAS Y ESTRATEGIAS DE MEDIACIÓN DEL APRENDIZAJE deben contribuir al desarrollo del conocimiento y a su construcción, al desarrollo del pensamiento y a la consolidación de valores.

EVALUACIÓN además de ser coherente con respecto a los objetivos, debe permitir verificar y valorar su cumplimiento. (MEP, 1994)

\subsubsection{Programa de Física}

El principal propósito del programa de Física es aportar elementos que se introducen con otras disciplinas para construir y reconstruir un concepto global de la naturaleza, desde los esquemas mentales que, haya formado cada estudiante acerca de su realidad.

La Física, desde el currículo, propicia la educación científica, y adquiere características propias del fenómeno educativo, de tal modo que la asignatura encuentra sus fundamentos en: 
La investigación, la cual hace de la Física una ciencia, según Goode (1983): De base, toda ciencia es un método (método científico), un modo de obtener conocimientos objetivos, precisos y sistemáticos acerca de la realidad; donde los procesos científicos permiten un ordenamiento en la búsqueda del conocimiento. (MEP, 2005)

La asignatura de Física forma parte del ciclo de Educación Diversificada, entre los fines de este ciclo se contempla la cantidad y profundidad de los contenidos que forman el perfil de salida, que está determinado por la manifestación de: "Habilidad para el ingreso a los estudios superiores y el desempeño, en la vida activa, de un papel adecuado en el nivel profesional correspondiente" (MEP, 2005).

Por medio del estudio de la Física se logra contribuir al desarrollo del pensamiento debido a las múltiples situaciones que se deben de afrontar para planear la solución de problemas variados.

El concepto de aprender nos lleva a un aspecto epistemológico del proceso de conocimiento definido por Pernudi (1988) como: "La capacidad del individuo para "aprender" las relaciones entre objetos de conocimiento, generados a partir de la interacción con el medio"; en el que este se desarrolle.

Al respecto Arauz y otros (1987) explican que esta concepción de conocimiento como proceso, plantea un problema epistemológico básico: el de las relaciones del sujeto cognoscente y el objeto de conocimiento, de modo que las interacciones recíprocas sujetoobjeto tienen como fin la adaptación, que es la inteligencia, en su acepción más dinámica. Se produce la necesidad de las personas de interactuar con el medio, lo que le provoca un desequilibrio. Estas reaccionan para recuperar el equilibrio utilizando la asimilación y la acomodación y así lograr la adaptación dinámica, que les permite conocer el medio y tratar de transformarlo. (MEP, 2005)

\subsubsection{Programa de Química}

El principal propósito educativo del programa de Química en la Educación Diversificada, consiste en que los estudiantes logren comprender la Química como una disciplina de 
estudio fundamental, por sus aportes al avance científico y tecnológico del mundo y así puedan entender los fenómenos químicos dados en la naturaleza.

La enseñanza de la Química no puede limitarse solo a dar información relativa a planteamientos teóricos de esta disciplina, sino también debe de ofrecer a los estudiantes la oportunidad de analizar situaciones de la vida cotidiana en los cuales se evidencian problemas que requieren de un conocimiento químico para su análisis y búsqueda de posibles explicaciones y soluciones. Será a partir de esta introducción al mundo de la Química como los estudiantes desarrollarán la sensibilidad para abordar las problemáticas ambientales, industriales, de salud y de alimentación. Además, se busca lograr que los jóvenes le den significado a los planteamientos teóricos de una disciplina con gran importancia en el desarrollo de la ciencia en nuestro tiempo y un gran potencial para el futuro desarrollo de la humanidad (MEP, 2005).

En el proceso de aprendizaje, los estudiantes logran identificar y caracterizar las distintas disciplinas científicas desarrolladas, para entender las relaciones entre los diferentes fenómenos dados en la naturaleza; así como las relaciones entre éstos y su importancia para los seres humanos. Es así como puede plantearse que la Química, como asignatura en la Educación Diversificada, es un medio para que los jóvenes logren comprender procesos y fenómenos de la naturaleza que les afectan de una u otra manera. La Química, como asignatura del plan de estudios de la Educación Diversificada, debe ofrecer a los jóvenes la posibilidad de desarrollar actitudes y valores propios del quehacer científico, gusto por explorar la naturaleza, sentido crítico en su relación con el medio y el uso de las nuevas tecnologías; así como la posibilidad de desarrollar un lenguaje que les facilite la producción y comprensión de diversos comunicados (orales y escritos) de carácter científico (MEP, 2005).

Considerando los principios fundamentales de la Química, los estudiantes podrán comprender mejor los procesos químicos que ocurren en la naturaleza y en ellos mismos, lo cual, les permitirá interactuar con su entorno natural. Con esta base los jóvenes estarán en capacidad de desarrollar las habilidades y destrezas necesarias para participar de los procedimientos del quehacer científico, base de la producción de nuevos conocimientos en el área de Química y que en el proceso de construcción de dichos conocimientos proveen los elementos fundamentales, con el fin de lograr una formación para un manejo racional de los 
productos químicos ofrecidos en el mercado; así como la capacidad para discernir acerca de la calidad de los productos de consumo diario (MEP, 2005).

Por lo tanto, el Programa de Química debe ser una guía curricular tanto para el docente como para el alumno, donde le ofrezca al estudiante la oportunidad para explorar, comprender y analizar desde el punto de vista científico y tecnológico el medio natural y sus implicaciones en la sociedad, permitiéndole un desarrollo más eficiente e integral tanto del sistema cognitivo como del psicomotor.

Por eso es importante que el alumno tenga experiencias de laboratorio como parte de su formación académica, donde pueda experimentar, conocer, aprender y aplicar sus experiencias y conocimientos previos a su formación.

En el contexto de este programa, se visualiza el laboratorio, no como el recinto equipado con material complejo, necesario en algunos casos, sino como oportunidades para que profesores y estudiantes analicen situaciones concretas, desde las cuales se identifiquen elementos valiosos, base para elaborar explicaciones conceptuales que ayuden a proponer soluciones a problemáticas específicas. Es importante en la organización de tales situaciones, como prácticas de laboratorio, aprovechar al máximo la utilización de recursos del medio para permitir a los estudiantes valorar la importancia de materiales comúnmente percibidos como inservibles o de un uso limitado. Estas vivencias le darán oportunidad para el desarrollo de la creatividad, en cuanto a la reutilización de materiales del medio. Otro recurso recomendable como experiencia de aprendizaje es el laboratorio "in situ", esto es, el análisis de situaciones tal y como se dan en la naturaleza o en el contexto donde residen los estudiantes. Existe una gran diversidad de procesos químicos llevados a cabo en el hogar, industrias de la comunidad, campos agrícolas y otros, se puede invitar a los estudiantes a analizarlos desde las perspectivas teóricas aportadas por el curso, o que se utilicen como base para la formulación de cuestionamientos a partir de los cuales se generará la teoría. (MEP, 2005)

Dentro del programa de Química se promueve en gran medida la utilización de laboratorios ambientales, y a su vez el estudio de las reacciones Químicas que suceden en nuestro hogar. Lo anterior con el objetivo de ejemplificar la presencia de la Química en la vida Volumen 8, Número 3, Año 2008, ISSN 1409-4703 
cotidiana, y de incentivar al estudiante, que el mundo que lo rodea, desde lo macro hasta lo micro, involucra a la química Química.

Gracias a los programas de estudio se logran alcanzar los ideales educativos de un pueblo, preparando a los futuros ciudadanos ante una sociedad tecnológica y a su vez a los futuros científicos de nuestro país.

\section{REFERENCIAS}

Bunge, Mario. (1984). Ciencia y desarrollo. Buenos Aires, Argentina: Ediciones siglo veinte.

Barnard J., Darrell. (1970). Enseñanza de las ciencias. Buenos Aires, Argentina: Librería del colegio.

Gurdián, Alicia. (1999). Política Social y Educación en Costa Rica (comp.). San José, Costa Rica: UNICEF.

Medina, Manuel y Kwiatkowsha, Teresa. (2000). Ciencia, tecnología/naturaleza, cultura en el siglo XXI. México: Anthropos.

Ministerio de Educación Pública - MEP, Costa Rica. (1994). Política educativa hacia el siglo XXI. San José, Costa Rica.

Ministerio de Educación Pública - MEP, Costa Rica. (2005). Programas de estudio de Física. Educación Diversificada. San José, Costa Rica.

Ministerio de Educación Pública - MEP, Costa Rica. (2005). Programas de estudio de Química. Educación Diversificada. San José, Costa Rica.

Morales, Orlando. (2001). Ciencia, tecnología y técnicas -ensayos-. San José, Costa Rica: INA

UNICEF. (1979). Nuevas tendencias en la enseñanza de las ciencias. Santiago de Chile: UNESCO. 